\title{
Comparison of the Effects of Acupressure and Electroacupuncture in Primary Dysmenorrhea: A Randomized Controlled Trial
}

\author{
Katayun Qorbanalipour ${ }^{1}$, Fariba Ghaderi ${ }^{1^{*}}{ }^{\mathbb{D}}$, Mohammad Asgari Jafarabadi $^{2}$
}

\begin{abstract}
Objectives: Dysmenorrhea or painful menstruation is a frequently observed fertility and femininity abnormality in women of childbearing age. Dysmenorrhea is treated by medical, complementary or surgical treatments. The aim of the present randomized clinical trial (RCT) study was to compare the effectiveness of electroacupuncture and acupressure treatment methods in reducing symptoms of primary dysmenorrhea.

Materials and Methods: In this double-blinded RCT, 64 female students who met the criteria were assigned into 2 groups (Intervention group: electroacupuncture and Control group: acupressure) using random allocation software (RAS). Pain according to visual analogue scale (VAS), menstrual distress severity according to Moos Menstrual Distress Questionnaire (MMDQ) and the frequency of drug use during menstruation were measured 3 times before and after treatment cycle and follow up cycle. Electroacupuncture and acupressure were done by applying electrical stimulation (ES) to needles or pressure at SP6 and SP4 points for 10 minutes on each side.

Results: The MMDQ $(P=0.000)$ and VAS $(P=0.000)$ showed a significant decrease in both groups but the rate of decline was higher in the electroacupuncture group.

Conclusions: According to the results, it was revealed that treatment with electroacupuncture can also be suggested as a treatment in complementary medicine for treatment of primary dysmenorrhea which has no reported side effects.

Keywords: Primary Dysmenorrhea, Acupuncture, Acupressure, Electroacupuncture, SP6 (Saninjiao), SP4 (Gongsun)
\end{abstract}

\section{Introduction}

Dysmenorrhea is painful menstruation that usually accompanied by cramping that is felt in the lower abdomen. Dysmenorrhea is generally divided into 2 types of primary and secondary. Primary dysmenorrhea is a spasmodic pain that is associated with ovulatory cycles, in the absence of demonstrable pathology. Secondary dysmenorrhea is a congestive pain that is associated with organic uterine or pelvic pathology such as endometriosis, adenomyosis, myomas and so on (1).

Dysmenorrhea can be accompanied by symptoms such as lower abdominal pain, diarrhea, nervousness, edema, nausea, headache, dizziness, fatigue, and so on (2-6).

Primary dysmenorrhea occurs when the uterus spasm starts because of decreasing blood supply of uterus. It is believed that the release of prostaglandins and other inflammatory mediators in the uterus is the main cause of primary dysmenorrhea $(3,7)$.

The prevalence of dysmenorrhea varies between \%18 to $\% 81$ based on different outcome measures in previous studies (8), but $10 \%$ of women suffer from severe symptoms of dysmenorrhea (5). Nowadays, dysmenorrhea is one of the most common causes of absenteeism in young women $(2,3,8-10)$. For example, in the United States, dysmenorrhea caused a loss of 600 million working hours a year and its economic loss is estimated to be 2 billion dollars a year (11). Previous studies in Iran showed the prevalence of $71 \%$ to $98.4 \%$ for primary dysmenorrhea among young Iranian women $(10,12)$.

Various treatments such as pharmacotherapy, complementary medicine, and surgery are suggested for dysmenorrhea $(13,14)$. Common pharmacotherapy includes NSAIDs (non-steroidal anti-inflammatory drugs) and oral contraceptives. Complementary treatments include dietary changes, herbal tea, the use of heat in the area, and Chinese medicine like acupressure, electroacupuncture, and acupuncture (5).

The Chinese believed that "chi" or vital energy flows in meridians and the specific points on meridians called acupoints affect certain organs of the human body. Acupressure is "energy balancing" and a type of "handson" techniques that balance energy flow, blood flow, lymphatic, hormonal and nerve conduction (13).

Chinese medicines believe that menstrual symptoms occur due to a lack of blood or blood stagnation or the existence of "chi" in the body. The main and the most effective acupuncture point in the gynecological conditions such as dysmenorrhea is spleen 6 or saninjiao 
(SP6) (Figure 1). This point is located at the confluence of 3 channels of the liver, kidney, and spleen (13). There are also another acupoints which are used for gynecological disorders: Bladder 47 (BL47), Bladder 23 (BL23), Spleen 9 (SP9), Spleen 10 (SP10), Spleen 4 (SP4) or Gongsun and so on. SP4 point is an effective point to reduce uterine spasm and contributes to regular menstrual bleeding (14). According to previous studies, acupressure is well known as a treatment method for dysmenorrhea $(2,15,16)$. In electroacupuncture, electrical stimulation (ES) is applied to needles inserted in acupoints (13).

Ren and Zhuang showed that electroacupuncture can improve blood circulation and hemodynamic in the uterus (17). Ma et al compared the effects of electroacupuncture at 3 points (SP6, GB39, and a nonmeridian point) in randomized clinical trial (RCT) and the results showed that electroacupuncture at point SP6 can immediately reduce the pain compared to point GB 39 and nonacupuncture point (14). In the same year, Wong conducted a study on 40 women suffered from dysmenorrhea in which an intervention group received acupressure at SP6 point and another group was advised to rest. In this study, a rapid and significant reduction was observed in VAS and MMDQ scale in the acupressure group (18). In another RCT, which was conducted in 2011 by Mohammad Alizadeh et al on 72 university students, acupressure at SP6 point was compared with control group (no treatment), the results showed acupressure is more effective in reducing symptoms of dysmenorrhea in both spasmodic and congestive pain types. Therefore, acupressure can be suggested to be used alone or in combination with other therapeutic methods for dysmenorrhea (6).

Yafang et al conducted a study on rats and showed that electroacupuncture reduces uterine artery spasm, which improves blood flow to the uterus and reduces pain. It showed that electroacupuncture at SP6 point is more effective compared to SP10 (19).

Acupressure and acupuncture, as relatively safe treatments with the least side effects, are suggested for treatment of dysmenorrhea (20), however, there are not many studies about the effects of electroacupuncture on dysmenorrhea. As we know electroacupuncture can increase the effects of acupuncture, so in the present study, we compared the effectiveness of electroacupuncture and acupressure on dysmenorrhea.

\section{Materials and Methods}

This study was a prospective double-blinded randomized clinical trial (RCT) which was conducted at the physiotherapy clinic of the faculty of Rehabilitation, Tabriz University of Medical Science, from January 2016 to July 2016.

A gynecologist visited the participants with self-reported primary dysmenorrhea. If the primary dysmenorrhea was definitely diagnosed, the participants who matched the inclusion and exclusion criteria were assigned randomly

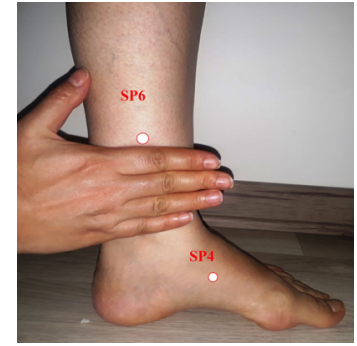

Figure 1. SP4 and SP6 Points

into 2 groups by random allocation software (RAS).

Virgin girls aged 18-26 years with a definite diagnosis of primary dysmenorrhea by a gynecologist and at least 2 years of regular menstruation between 21 to 35 days were included in this study.

Patients with secondary dysmenorrhea or any history of pelvic organs surgery or any types of itching, and abnormal secretions or using any other drugs for specific health problems such as mental disorders or any other problems or experiencing any stressful condition such as parental separation or death of close relatives in the past six months or a history of smoking or alcohol use were excluded.

The intervention group received electroacupuncture $(\mathrm{n}=31)$ and the control group received acupressure $(n=33)$ during the experimental cycle and both techniques were applied by a trained therapist.

Measured outcomes included pain severity according to visual analogue scale (VAS), menstrual distress severity based on the Moos Menstrual Distress Questionnaire (MMDQ) and the frequency of drug use during menstruation. The validated Persian version of the MMDQ was used in this study (21).

For baseline data of each participant, the VAS and MMDQ questionnaires were completed in the first 3 days of the first period. The participants received their own treatment (acupuncture or electroacupuncture in the second cycle and the third cycle was follow up cycle. The assessor and statistical analyzer were blinded.

\section{Control Group: Acupressure}

Acupressure was applied to SP4 and SP6 for 10 minutes, 5 minutes in a clockwise direction and 5 minutes in a counter clockwise direction in each limb (6).

Intervention Group: Electroacupuncture

Electroacupuncture was done by inserting needles into SP6 and SP4 points for 10 minutes (Figure 1). ES was done by electroacupuncture unit (ES 160, Japan, ITO Company). A square shaped stimulation with a frequency of $6 \mathrm{~Hz}$ and 1.5-second intervals and tolerable intensity was applied.

In both groups, in order to prevent uncontrolled use of drugs, a specified number of painkillers (5 ibuprofen tablets from Hakim pharmaceutical company) were given to the volunteers (6) to use in case of unbearable pain and 
report it. Finally, the unused pills were collected.

VAS and MMDQ were assessed in the first 3 days of 3 consecutive menstrual cycles. In the first cycle, the days with the most pain in menstruation were identified for each person and the severity of baseline symptoms (assessment cycle) was assessed. The second cycle was the intervention cycle and the third cycle was the follow-up cycle.

\section{Statistical Analysis}

In order to analyze the data, SPSS software version 21.0 was used. The repeated measures were used for intra-group analysis and if the result of the repeated measure was significant, Sidak test was needed. When the variable did not have a normal distribution, nonparametric Man-Whitney test was used to determine the differences between groups. When the variable was normally distributed considering continuous variables, independent $t$ test was used and for nominal and ordinal variables, chi-square test was used. $P$ value $<0.05$ was considered significant.

\section{Results}

The baseline characteristics of the 2 groups were compared considering the variables of age, body mass index (BMI), menstrual cycle, menstrual regularity, physical activity, family socioeconomic level, experience of stress, and participants' education level using independent $t$ test. The results showed that there were no significant differences between 2 groups before intervention $(P>0.005)$ except for their level of education $(P=0.006)$, so the results were adjusted for the level of education in 2 groups (Table 1 ).

The results of the repeated measures and Sidak showed that both intervention methods significantly reduced the severity of pain and distress in dysmenorrhea during intervention cycle and follow up cycle (Table 2), but MMDQ had more reduction in electroacupuncture group in comparison with acupressure group (Figure 2).

VAS decreased in both groups and there was no statistical difference between 2 groups in the intervention cycle or follow up cycle (Figure 3 ).

Considering the criteria of frequency of drug use which is an ordinal variable, Man-Whitney test was used in the first, second, and third months and it was found that there was no significant difference between the 2 groups.

\section{Discussion}

The results showed that both VAS and MMDQ decreased significantly in 2 intervention and control groups, but the rate of decline was higher in electroacupuncture group.

It was the first time that the day of the most severe symptoms for each participant was determined and the treatment protocol was done on that day.

Gharloghi et al evaluated the average menstrual pain in the first 3 days of the first cycle and in the second cycle, the intervention was applied once per day in those days of the second cycle (9). Wong et al assessed pain severity and symptoms in first cycle but just used the average of them

Table 1. Descriptive Statistics of Demographic Characteristics ${ }^{a}$

\begin{tabular}{|c|c|c|c|c|}
\hline & & $\begin{array}{c}\text { Control ( } n=33 \text { ) } \\
\text { Mean (SD) }\end{array}$ & $\begin{array}{c}\text { Experimental }(n=31) \\
\text { Mean (SD) }\end{array}$ & Sig. (2-tailed) \\
\hline Age & & $23.67(3.01)$ & $22.94(2.85)$ & 0.324 \\
\hline BMI & & $22.88(2.85)$ & 23.04 (3.24) & 0.843 \\
\hline VAS & & $5.49(1.21)$ & $5.02(1.83)$ & 0.068 \\
\hline MMDQ & & $23.31(3.53)$ & 26.09 (6.39) & 0.057 \\
\hline \multirow{2}{*}{ Experience stress } & Yes & $3(9.1)$ & $8(25.8)$ & \multirow{2}{*}{0.103} \\
\hline & No & $30(90.9)$ & $23(74.2)$ & \\
\hline \multirow{4}{*}{ Educational level } & BSc Student & $11(33.3)$ & $21(67.7)$ & \multirow{4}{*}{0.006} \\
\hline & MSc student & $6(18.2)$ & $1(3.2)$ & \\
\hline & Graduated & $12(36.4)$ & $8(25.8)$ & \\
\hline & MD student & $4(12.1)$ & $1(3.2)$ & \\
\hline \multirow{4}{*}{ Parent salary } & Excellent & $8(24.2)$ & $5(16.1)$ & \multirow{4}{*}{0.085} \\
\hline & Good & $9(27.3)$ & $16(51.6)$ & \\
\hline & Mean & $13(39.4)$ & $10(32.3)$ & \\
\hline & Poor & $3(9.1)$ & $0(0.0)$ & \\
\hline \multirow{2}{*}{ Regular menstruation cycle } & Yes & $32(97.0)$ & $26(89.3)$ & \multirow{2}{*}{0.075} \\
\hline & No & $1(3.0)$ & $5(16.1)$ & \\
\hline \multirow{5}{*}{ Cycle length (days) } & $<21$ & $0(0.00)$ & $3(9.7)$ & \multirow{5}{*}{0.095} \\
\hline & $21-26$ & $15(45.5)$ & $12(38.7)$ & \\
\hline & $26-31$ & $10(30.3)$ & $7(22.6)$ & \\
\hline & $31-35$ & $7(21.2)$ & $9(29.0)$ & \\
\hline & $>35$ & $1(3.0)$ & $0(0.00)$ & \\
\hline \multirow{2}{*}{ Doing exercise } & Yes & $8(25.8)$ & $9(27.3)$ & \multirow{2}{*}{0.221} \\
\hline & No & $23(47.2)$ & $24(72.7)$ & \\
\hline
\end{tabular}

${ }^{a}$ According to independent $t$ test. 
Table 2. Analytical Statistics of Primary Outcomes in 3 Cycles $^{a}$

\begin{tabular}{|c|c|c|c|c|c|}
\hline & \multicolumn{2}{|c|}{ Electroacupuncture $(n=31)$} & \multicolumn{2}{|c|}{ Acupressure $(n=33)$} & \multirow{2}{*}{$P$ Value } \\
\hline & [Range] & Mean (SD) & [Range] & Mean (SD) & \\
\hline VAS before & [1.6-8.0] & $5.02(1.83)$ & {$[3.0-8.3]$} & $5.49(1.21)$ & 0.068 \\
\hline VAS at time & {$[.33-7.0]$} & $3.78(1.84)$ & {$[2.6-6.3]$} & $4.43(1.04)$ & 0.00 \\
\hline VAS after & {$[0.0-6.3]$} & $3.38(1.85)$ & {$[1.3-6.6]$} & $4.01(1.21)$ & 0.00 \\
\hline$P$ value (intragroup) & \multicolumn{2}{|c|}{0.00} & \multicolumn{2}{|c|}{0.00} & \\
\hline MMDQ before & {$[19.0-49.6]$} & $26.09(6.39)$ & [18. 3-36.6] & $23.31(3.53)$ & 0.057 \\
\hline MMDQ at time & {$[17.0-35.3]$} & $22.65(4.03)$ & {$[17.0-36.6]$} & $21.49(3.53)$ & 0.00 \\
\hline MMDQ after & {$[16.3-29.6]$} & $21.37(3.42)$ & {$[16.3-34.6]$} & $20.19(3.16)$ & 0.00 \\
\hline$P$ value (intragroup) & \multicolumn{2}{|c|}{0.00} & \multicolumn{2}{|c|}{0.00} & \\
\hline
\end{tabular}

${ }^{a}$ According to repeated measures and Sidak.

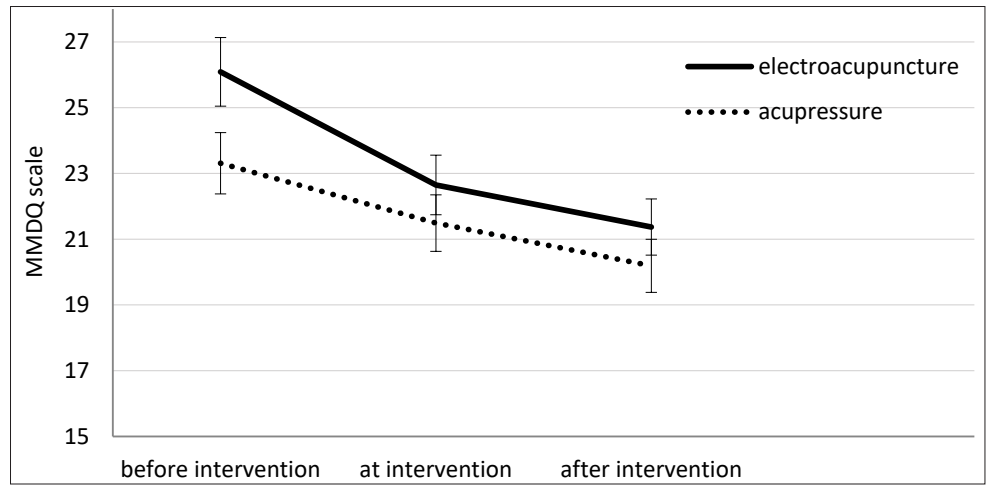

Figure 2. MMDQ Scales in 2 Treatment Groups in 3 Cycles (according to repeated measures and Sidak).



Figure 3. MVAS Scales in 2 Treatment Groups in 3 Cycles (according to repeated measures and Sidak).

and in next menstruation cycle, the intervention was done on the first day of menstruation (18)

The results of this study were in line with the results of a study by Wong et al who revealed that acupressure has long lasting and cumulative effects in reducing the pain of primary dysmenorrhea. They also showed that acupressure at SP6 can significantly decrease pain immediately and it lasted 3 cycles after intervention (18). Moreover, Gharlogi et al showed that acupressure at SP6 and SP8 points significantly reduced the severity of dysmenorrhea immediately, 30 minutes, 1 hour, and 2 hours after intervention (9). Furthermore, MirbagherAjorpaz et al showed that applying acupressure at SP6 significantly reduced dysmenorrheal pain immediately 
after intervention and after 1, 2, and 3 hours (22).

Researchers suggested that manipulating the small myelinated peripheral nerve fibers in muscles with needles can transmit signals to central nervous system (CNS) resulting in the release of substances such as enkephalin, norepinephrine and so on, which alleviates pain (9). Acupressure is the stimulation of acupoints and meridians without the use of needles, and probably has other effects such as reducing tension, promoting wellness, and providing comfort (10) but these outcomes have not been measured in the present study.

Ma et al reported that applying electroacupuncture at SP6 is an important clinical point in dysmenorrheal pain and electroacupuncture at this point, compared with the other points, reduces VAS scale rapidly (14).

Yafang et al Showed that electroacupuncture not only reduces the symptoms of dysmenorrhea but also caused a significant improvement in uterine blood flow and hemodynamics (19).

Some researchers such as Ren and Zhuang (17) and Yafang (19) believe that stimulating acupressure points by electro-acupuncture can reduce spasticity of the uterine arteries, improve blood flow and finally reduce menstrual pain and symptoms; this claim requires more microscopic examination.

Stimulating the acupoints causes a particular afferent activity pattern in peripheral nerve fibers. low-frequency electroacupuncture caused neuropeptide release in the CNS, which induced functional changes in organs (23). It seems that the existence of 2 factors including reduction of spasticity of the uterine arteries and release of neuropeptides can reduce menstrual pain and symptoms.

In this study, electroacupuncture could relieve the pain and distress better than acupressure, which is similar to other studies but because of the pain in inserting needles, it may be used in cases with severe dysmenorrhea.

\section{Strengths and Limitations}

The main and notable superiority of this study is determining the days with the most pain in menstruation for each participant in assessment cycle and the intervention was applied on the same day for each participant in the second cycle.

The possibility of treatment use in acupressure treatment group especially in the third month was one of the limitations in this study as individuals had learned the process to some extent. However, the researcher had to rely on individuals' verbal commitment to avoid using treatment in control month.

\section{Conclusions}

This study revealed that electroacupuncture treatment can be an effective method for treating primary dysmenorrhea and it was more effective than acupressure in reducing distress in primary dysmenorrhea and has long-term effects for at least 1 month. In addition, in line with the previous studies, in this study, there were no negative side effects for electroacupuncture. According to these findings, we can consider electroacupuncture as a safe treatment for primary dysmenorrhea, especially in severe cases.

\section{Conflict of Interests}

Authors declare that they have no conflict of interests.

\section{Ethical Issues}

This study was approved by the Ethics Committee of Tabriz University of Medical Science and it was registered in Iranian Registry of Clinical Trials (identifier: IRCT2015012518760N2).

\section{Financial Support}

Research Vice-Chancellor of Tabriz University of Medical Sciences.

\section{Acknowledgements}

Thanks to our colleagues Maryam Ghodrati and Safi Khanlou and other students of Tabriz University who helped us conduct this research.

\section{References}

1. Lin JA, Wong CS, Lee MS, et al. Successful treatment of primary dysmenorrhea by collateral meridian acupressure therapy. J Manipulative Physiol Ther. 2010;33(1):70-75. doi:10.1016/j.jmpt.2009.11.003

2. Jiang HR, Ni S, Li JL, et al. Systematic review of randomized clinical trials of acupressure therapy for primary dysmenorrhea. Evid Based Complement Alternat Med. 2013;2013:169692. doi:10.1155/2013/169692

3. Deb S, Raine-Fenning N. Dysmenorrhoea. Obstet Gynaecol Reprod Med. 2008;18(11):294-299. doi:10.1016/j. ogrm.2008.08.007

4. Chung YC, Chen HH, Yeh ML. Acupoint stimulation intervention for people with primary dysmenorrhea: Systematic review and meta-analysis of randomized trials. Complement Ther Med. 2012;20(5):353-363. doi:10.1016/j. ctim.2012.02.008

5. Chen MN, Chien LW, Liu CF. Acupuncture or Acupressure at the Sanyinjiao (SP6) Acupoint for the Treatment of Primary Dysmenorrhea: A Meta-Analysis. Evid Based Complement Alternat Med. 2013;2013:493038. doi:10.1155/2013/493038

6. Charandabi SM, Nashtaei MS, Kamali S, Majlesi R. The effect of acupressure at the Sanyinjiao point (SP6) on primary dysmenorrhea in students resident in dormitories of Tabriz. Iran J Nurs Midwifery Res. 2011;16(4):309-317.

7. Saadatabadi FS, Bambaichi E, Esfarjani F. Effect of Six Weeks Flexibility Training on Dysmenorrhea. Journal of Isfahan Medical School. 2010;28(109):1-7.

8. Witt CM, Reinhold T, Brinkhaus B, Roll S, Jena S, Willich SN. Acupuncture in patients with dysmenorrhea: a randomized study on clinical effectiveness and cost-effectiveness in usual care. Am J Obstet Gynecol. 2008;198(2):166.e161168. doi:10.1016/j.ajog.2007.07.041 
9. Gharloghi S, Torkzahrani S, Akbarzadeh AR, Heshmat R. The effects of acupressure on severity of primary dysmenorrhea. Patient preference and adherence. 2012;6:137. doi: 10.2147/PPA.S27127.

10. Shabani Nashtaei M, Mohammadalizadeh S. Primary dysmenorrhea among dormitory students of Tabriz. Nurs Midwifery J. 2010;5(18):15-21.

11. Kokjohn K, Schmid DM, Triano JJ, Brennan PC. The effect of spinal manipulation on pain and prostaglandin levels in women with primary dysmenorrhea. J Manipulative Physiol Ther. 1992;15(5):279-285.

12. Ghaderi F, Asghari Jafarabadi M, Mohseni Bandpei MA. Dysmenorrhea and self-care strategies in Iranian female students: a regression modeling of pain severity and underlying factors. Int J Adolesc Med Health. 2016;29(6). doi:10.1515/ijamh-2016-0017

13. Wang MC, Hsu MC, Chien LW, Kao CH, Liu CF. Effects of auricular acupressure on menstrual symptoms and nitric oxide for women with primary dysmenorrhea. J Altern Complement Med. 2009;15(3):235-242. doi:10.1089/ acm.2008.0164

14. Ma YX, Ma LX, Liu XL, et al. A comparative study on the immediate effects of electroacupuncture at Sanyinjiao (SP6), Xuanzhong (GB39) and a non-meridian point, on menstrual pain and uterine arterial blood flow, in primary dysmenorrhea patients. Pain Med. 2010;11(10):1564-1575. doi:10.1111/j.1526-4637.2010.00949.x

15. Chen YW, Wang HH. The effectiveness of acupressure on relieving pain: a systematic review. Pain Manag Nurs. 2014;15(2):539-550. doi:10.1016/j.pmn.2012.12.005

16. Han JS. Acupuncture: neuropeptide release produced by electrical stimulation of different frequencies. Trends Neurosci. 2003;26(1):17-22. doi:10.1016/S01662236(02)00006-1

17. Ren R, Zhuang LX. Impact of electroacupuncture on hemorrheology and uterine arteries hemodynamics in treating primary dysmenorrhea. Chinese Archives of Traditional Chinese Medicine. 2010;(3):649-651

18. Wong CL, Lai KY, Tse HM. Effects of SP6 acupressure on pain and menstrual distress in young women with dysmenorrhea. Complement Ther Clin Pract. 2010;16(2):64-69. doi:10.1016/j.ctcp.2009.10.002

19. Yafang Z, Chunhua L, Bo J. Effect of Electroacupuncture at Sanyinjiao and Xuehai on Uterine Microcirculation in Dysmenorrhea Rats. Chinese Journal of Microcirculation. 2011;2:004.

20. Ernst E, Lee MS. Acupressure: an overview of systematic reviews. J Pain Symptom Manage. 2010;40(4):e3-7. doi:10.1016/j.jpainsymman.2010.07.003

21. Qorbanalipour K, Ghaderi F, Asghari Jafarabadi M, Mohammadalizade Charandabi S. Validity and reliability of the Persian version of modified Moos Menstrual Distress Questionnaire. The Iranian Journal of Obstetrics, Gynecology and Infertility. 2016;19(29):11-18.

22. Mirbagher-Ajorpaz N, Adib-Hajbaghery M, Mosaebi F. The effects of acupressure on primary dysmenorrhea: a randomized controlled trial. Complement Ther Clin Pract. 2011;17(1):33-36. doi:10.1016/j.ctcp.2010.06.005

23. Stener-Victorin E, Wu X. Effects and mechanisms of acupuncture in the reproductive system. Auton Neurosci. 2010;157(1-2):46-51. doi:10.1016/j.autneu.2010.03.006

(c) 2018 The Author (s); This is an open-access article distributed under the terms of the Creative Commons Attribution License (http://creativecommons.org/licenses/by/4.0), which permits unrestricted use, distribution, and reproduction in any medium, provided the original work is properly cited. 